\title{
More productive in vitro culture of Cryptosporidium parvum for better study of the intra- and extracellular phases
}

\author{
G Perez Cordón, C Marin, D Romero, C Rosales, M Sánchez Moreno, \\ MJ Rosales ${ }^{+}$
}

Departamento de Parasitologia, Facultad de Ciencias, Instituto de Biotecnología, 18071, Universidad de Granada, España

The great difficulties in treating people and animals suffering from cryptosporidiosis have prompted the development of in vitro experimental models. Due to the models of in vitro culture, new extracellular stages of Cryptosporidium have been demonstrated. The development of these extracellular phases depends on the technique of in vitro culture and on the species and genotype of Cryptosporidium used. Here, we undertake the molecular characterization by polymerase chain reaction-restriction fragment lenght polymorphism of different Cryptosporidium isolates from calves, concluding that all are C. parvum of cattle genotype, although differing in the nucleotide at positions 472 and 498. Using these parasites, modified the in vitro culture technique for HCT-8 cells achieving greater multiplication of parasites. The HCT-8 cell cultures, for which the culture had not been renewed in seven days, were infected with C. parvum sporozoites in RPMI-1640 medium with 10\% IFBS, CaCl 2 and $\mathrm{MgCl}_{2} 1 \mathrm{mM}$ at $\mathrm{pH}$ 7.2. Percentages of cell parasitism were increased with respect to control cultures (71\% at 48 h vs 14.5\%), even after two weeks (47\% vs 1.9\%). Also, the percentage of extracellular stages augmented $(25.3 \%$ vs $1.1 \%$ at $96 \mathrm{~h})$. This new model of in vitro culture of C. parvum will enable easier study of the developmental phases of $\mathrm{C}$. parvum in performing new chemotherapeutic assays.

Key words: Cryptosporidium - in vitro - culture - extracellular stages

Cryptosporidium infections have been reported in human and in most domestic animals. Most infections have been described in mammals and are attributed to bovine sources of $C$. parvum (Ramirez et al. 2004). Cattle have different species of Cryptosporidium: C. pestis (bovine genotype of $C$. parvum), C. bovis, $C$. andersoni, and Cryptosporidium "deer-like genotype" (Slapeta 2006).

Although Slapeta (2006) proposed the re-classification of $C$. parvum as $C$. pestis, still exists controversy about this. The cattle genotype of $C$. parvum can infect other mammals, including human, and has been responsible of waterborne outbreaks as well as outbreaks among school children associated with farm visits (Monis \& Thomson 2003, Becher et al. 2004).

It is known that different Cryptosporidium isolates from various regions have different antigens, virulence, infectivity, and sensitivity to drugs as well as disinfectants (McDonald et al. 1991, Griffin et al. 1992, Fayer et al. 2000). Therefore, it is necessary to characterize the parasites molecularly in each region and know the parasites to be used in the in vitro culture.

The first complete in vitro culture of $C$. parvum mentioning the appearance of extracellular stages was performed by Rosales et al. (1993) in cells Madin-Darby canine kidney MDCK cells. Afterwards, different cell lines were used as bovine fallopian tube epithelial cells

Corresponding autor: mjrosale@ugr.es

Received 16 November 2006

Accepted 20 June 2007
(Yang et al. 1996), Caco-2, HT29, and HCT-8 (Maillot et al. 1997, Hijjawi et al. 2001, Hijjawi 2003), VELI cells (Lacharme et al. 2004), and Hijjawi et al. (2004) described in vitro development of $C$. parvum in host cellfree culture.

Studies by Carreno et al. (1999), based on SSrRNA sequence, and Leander et al. (2003), based on SSU rDNA and $\beta$-tubulin sequences showed that the gregarines and Cryptosporidium formed a clade separate from the other major apicomplexa clade containing the coccidian. Hijjawi et al. $(2002,2004)$ reported the presence of extracellular gamont-like stages in the cycle of $C$. parvum and $C$. andersoni with HCT-8 cells or in RPMI1640 maintenance medium devoid of host cells. Recently, Rosales et al. (2005) has confirmed, by optical, Nomarski, and transmission electron microscopy images, the existence of extracellular trophozoite/gamont, stages in syzygy, zygotes, and spores with eight sporozoites during the in vitro culture of $C$. parvum on MDCK, HCT-8, and Vero cells as well as alveolar macrophages. It appears that, according to the methodology used in the in vitro culture and the Cryptosporidium species/ genotype used, some extracellular phases or others are developed. These extracellular stages were few in number in all the reports, and thus it was considered necessary to review the culture technique for better study (Hijjawi et al. 2002, 2004, Rosales et al. 2005).

In the present work, we undertake molecular characterization, using the polymerase chain reaction-restriction fragment (PCR-RFLP), of different Cryptosporidium isolates from calves, and we assay different factors in order to develop better technique of in vitro culture for HCT-8 cells, with the aim of studying the different phases (intra- and extracellular) of the biological cycle of Cryptosporidium with a more productive culture. 


\section{MATERIALS AND METHODS}

Molecular characterization of Cryptosporidium species and genotypes - A total of 120 faecal samples were collected from calves of less than 21 days old from two dairy farms of Granada (Spain). All the samples were diarrheic. C. parvum oocysts were purified on a potassium bromide discontinuous gradient (Entrala et al. 2000). The parasites were stored in PBS at $4^{\circ} \mathrm{C}$ until use.

All the faecal samples with Cryptosporidium, n 17, were processed for molecular characterization of Cryptosporidium species and genotypes.

DNA extraction and molecular characterization was done by PCR-RFLP according to Pérez-Cordón et al. (2005). Oocysts were digested using the technique of Robertson et al. (1993). Genomic DNA was extracted with phenol/choloroform/isoamyl alcohol as Ward et al. (2001). Cryptosporidium species and genotypes were determined by PCR of an SSUrRNA gene fragment and RFLP analysis as previously described (Xiao et al. 1999 a,b) but using the endonucleases SspI and VspI.

The second PCR product of 819 to $837 \mathrm{pb}$ was sequenced by Sistemas Genómicos, S.L. (Valencia, Spain) by single-strand sequencing of both ends of the insert performed with the amplification primers used during the PCR. Specific primers were designed from the sequences obtained during the previous sequencing phase (primer walking) and this step was repeated until one whole contig could be attained. Afterwards, the annealing and assembly of all the sequences obtained during the sequencing phase and contig editing was performed. Editing, deletion of all cloning vector sequences and settlement of all possible undetermined nucleotides was carried out.

This sequence was confirmed by the program Needle (Neddleman-Wunsch global alignment), with the nucleotide sequences from the ribosomal RNA gene published in the database GenBank (AF093490).

Modifications to the in vitro culture of C. parvum on cells - The in vitro culture of $C$. parvum on human ileocoecal adenocarcinoma (HCT 8) followed the methodology of Rosales et al. (1993) with different modifications: (1) the cell cultures were infected with sporozoites of $C$. parvum in RPMI-1640 medium with different concentrations of inactivated fetal bovine serum (IFBS), 2.5, 5, and 10\%, and the cultures were maintained with this medium; (2) $\mathrm{CaCl}_{2}$ and $\mathrm{MgCl}_{2} 1 \mathrm{mM}$ were added to the culture medium; (3) cell cultures were infected with sporozoites in RPMI-1640 at different $\mathrm{pH}$ values $(7.4,7.7,8.0,8.5)$; (4) the HCT-8 cells in culture medium that had not been renewed in seven days (i.e. nutrient depleted) were infected.

All the cell cultures infected were maintained for two weeks. Cultures were observed with Nomarski and light microscope (100x) every $24 \mathrm{~h}$ and interrupted at $48 \mathrm{~h}$ and at two weeks by fixation with methanol, whereupon they were stained with alcian-blue and Giemsa (Rosales et al. 1994) for observation with light microscopy to calculate the percentage of parasitized cells. About 1000 cells were counted.

After the analysis of the results, the best conditions for the development of the parasite were selected and an overall experiment was conducted with the following technique: the HCT-8 cell cultures for which the culture had not been renewed in seven days were infected with C. parvum sporozoites in RPMI-1640 medium with $10 \%$ IFBS, $\mathrm{CaCl}_{2}$, and $\mathrm{MgCl}_{2} 1 \mathrm{mM}$ at $\mathrm{pH}$ 7.2. These cultures were maintained two weeks in the same culture medium and examined every $24 \mathrm{~h}$ under the Nomarski and light microscope. For the evaluation of parasite number and stage differentiation, some of the cultures were interrupted at 48, 72, 96, and two weeks. The glass disks with the infected cells were removed, fixed with methanol, and then stained with alcian-blue and Giemsa (Rosales et al. 1994) for observation with light microscopy. All the cells in each microwell were counted and the percentage of parasitized cells and of different $C$. parvum development stages were calculated. Supernatant of the infected culture was centrifuged at $1000 \mathrm{~g}$ for $5 \mathrm{~min}$ and extracellular stages were observed and counted with Nomarski microscopy.

All the experiments were conducted four times and in all cases control HCT-8 cells were infected with $C$. parvum sporozoites following the technique of Rosales et al. (1993).

Independent-sample T-test was applied in order to compare the percentage of parasitism of experimental and control conditions.

\section{RESULTS AND DISCUSSION}

The sequencing of the second product of the 834-pb PCR of all the samples was identical. All the faecal samples studied by PCR-RFLP contained the same species of Cryptosporidium. This sequence (Fig. 1b) presented $99.8 \%$ similarity with the C. parvum sequence of cattle genotype, published in GenBanK AF093490 (Fig. 1a). Differences in the nucleotides appeared only at positions 472 and 498 (Fig. 1b underlined).

After the parasites were identified, a series of modifications were made in the in vitro culture technique of Rosales et al. (1993) with the aim of achieving more productive cultures for better study of the extracellular stages of $C$. parvum, which have been few in number in all previous the studies (Rosales et al. 1993, 2005, Hijjawi et al. 2002, 2004), and for new chemotherapeutic assays. In 1993, we indicated the existence of extracellular forms in the in vitro culture of $C$. parvum on MDCK cells (Rosales et al. 1993). This finding was supported afterwards by Hijjawi et al. (2002, 2004). The in vitro culture technique of C. parvum of Rosales et al. (1993) enabled the observation of the different extracellular stages, such as trophozoites/gamont, stages in syzygy, zygotes, and spores with eight sporozoites, as demonstrated several years later (Rosales et al. 2005). These developmental phases appeared in the supernatant of the cultures with all the cells that had been assayed (MDCK, HCT 8, Vero, alveolar macrophages), although the number rose in the case of the HCT-8 cells, as the percentage of the rest of the $C$. parvum developmental phases also increased. On the other hand, Hijjawi et al. (2002, 2004) detected only gamont-like extracellular stages, using HCT-8 cells or in host cell-free cultures. The appearance of more extracellular phases following the methodology of Rosales et al. (1993) than with that of Hijjawi et al. $(2002,2004)$ may be due to the use of excystation techniques and different cultures or else to the different composition of RPMI-1640 medium used 
a 1 GGAAGGGTTGTATTTATTAGATAAAGAACCAATATAATTGGTGACTCATA 50 b 1 GGAAGGGTTGTATTTATTAGATAAAGAACCAATATAATTGGTGACTCATA 50

a 51 ATAACTTTACGGATCACATTAAATGTGACATATCATTCAAGTTTCTGACC 100 b 51 ATAACTTTACGGATCACATTAAATGTGACATATCATTCAAGTTTCTGACC 100 a 101 TATCAGCTTTAGACGGTAGGGTATTGGCCTACCGTGGCAATGACGGGTAA 150 b 101 TATCAGCTTTAGACGGTAGGGTATTGGCCTACCGTGGCAATGACGGGTAA 150 a 151 CGGGGAATTAGGGTTCGATTCCGGAGAGGGAGCCTGAGAAACGGCTACCA 200 b 151 CGGGGAATTAGGGTTCGATTCCGGAGAGGGAGCCTGAGAAACGGCTACCA200 a 201 CATCTAAGGAAGGCAGCAGGCGCGCAAATTACCCAATCCTAATACAGGGA250 b 201 CATCTAAGGAAGGCAGCAGGCGCGCAAATTACCCAATCCTAATACAGGGA250

a 251 GGTAGTGACAAGAAATAACAATACAGGACTTTTTGGTTTTGTAATTGGAA 300 b 251 GGTAGTGACAAGAAATAACAATACAGGACTTTTTGGTTTTGTAATTGGAA 300 a 301 TGAGTTAAGTATAAACCCCTTTACAAGTATCAATTGGAGGGCAAGTCTGG 350 b 301 TGAGTTAAGTATAAACCCCTTTACAAGTATCAATTGGAGGGCAAGTCTGG 350 a 351 TGCCAGCAGCCGCGGTAATTCCAGCTCCAATAGCGTATATTAAAGTTGTT 400 b 351 TGCCAGCAGCCGCGGTAATTCCAGCTCCAATAGCGTATATTAAAGTTGTT 400

a 401 GCAGTTAAAAAGCTCGTAGTTGGATTTCTGTTAATAATTTATATAAAATA 450 b 401 GCAGTTAAAAAGCTCGTAGTTGGATTTCTGTTAATAATTTATATAAAATA 450

a 451 TTTTGATGAATATTTATATAATATTAACATAATTCATATTACTATATATT 500 b 451 TTTTGATGAATATTTATATAACATTAACATAATTCATATTACTATATNTT 500 a 501 TTAGTATATGAAATTTTACTTTGAGAAAATTAGAGTGCTTAAAGCAGGCA 550 b 501 TTAGTATATGAAATTTTACTTTGAGAAAATTAGAGTGCTTAAAGCAGGCA 550 a 551 TATGCCTTGAATACTCCAGCATGGAATAATATTAAAGATTTTTATCTTTC 600 b 551 TATGCCTTGAATACTCCAGCATGGAATAATATTAAAGATTTTTATCTTTC 600 a 601 TTATTGGTTCTAAGATAAGAATAATGATTAATAGGGACAGTTGGGGGCAT 650 b 601 TTATTGGTTCTAAGATAAGAATAATGATTAATAGGGACAGTTGGGGGCAT 650 a 651 TTGTATTTAACAGTCAGAGGTGAAATTCTTAGATTTGTTAAAGACAAACT 700 b 651 TTGTATTTAACAGTCAGAGGTGAAATTCTTAGATTTGTTAAAGACAAACT 700

a 701 AATGCGAAAGCATTTGCCAAGGATGTTTTCATTAATCAAGAACGAAAGTT 750 b 701 AATGCGAAAGCATTTGCCAAGGATGTTTTCATTAATCAAGAACGAAAGTT 750

a 751 AGGGGATCGAAGACGATCAGATACCGTCGTAGTCTTAACCATAAACTATG 800 b 751 AGGGGATCGAAGACGATCAGATACCGTCGTAGTCTTAACCATAAACTATG 800

a 801 CCAACTAGAGATTGGAGGTTGTTCCTTACTCCTT 834 b 801 CCAACTAGAGATTGGAGGTTGTTCCTTACTCCTT 834

Fig. 1: sequence of 834 bases pair of the $18 \mathrm{~S}$ small subunit (SSU) rRNA gene locus of Cryptosporidium obtained from the secondary polymerase chain reaction step. a: sequence from GenBank AF093490; b: sequence of isolates from cattle (Granada, Spain) with the different oligonucleotides underlined.

during the culture. In the cultures of Hijjawi et al. (2002, 2004) bovine bile, glucose, and ascorbic acid were added to the RPMI-1640 medium, and some of the these compounds could limit the development of some extracellular stages of C. parvum.

In this work, calf genotype $C$. parvum was cultured on HCT-8 cells and different modifications of the RPMI1640 culture medium were made during the infection of HCT-8 cells with $C$. parvum sporozoites and during the parasite-cell interaction (two weeks). Thus, changes in $\mathrm{pH}$ and different IFBS concentrations were assayed, $\mathrm{CaCl}_{2}, \mathrm{MgCl}_{2}$ were added to the culture medium, and cells were subjected to nutrient depletion. The results (Table I) reflect that the most effective approach was to infect cells cultured in nutrient-depleted RPMI-1640 medium. The nutrient depleted cells were infected more easily with the $C$. parvum sporozoites in the fresh culture medium, parasitism percentages being $65 \%$ and control $15.2 \%$. Also, the parasitism percentages increased when $\mathrm{CaCl}_{2}$ was added to the medium (23.2 vs $15.5 \%$ in control) and somewhat more with $\mathrm{MgCl}_{2}$ (30 vs $15.3 \%$ control), good results also being achieved in the maintenance of the continuous culture for two weeks (24.5 vs $4.3 \%$ ). As percentages of cell parasitism were increased, extracellular stages of $C$. parvum augmented too. This was not surprising, as the calcium and magnesium at a concentration of $1 \mathrm{mM}$ in the form of $\mathrm{CaCl}_{2}$ and $\mathrm{MgCl}_{2}$ increased the mobility of Toxoplasma gondii sporozoites and C. parvum (Hamer et al. 1994). Changes in $\mathrm{pH} 7.4,7.7,8$ or 8.5 demonstrated that the most effective was $\mathrm{pH} 7.2$ of the controls as Rosales et al. (1993). 
TABLE I

Percentage of parasitism of Cryptosporidium parvum in HCT 8 cells under different conditions ${ }^{a}$

\begin{tabular}{|c|c|c|c|c|c|c|c|c|c|}
\hline \multirow{3}{*}{$\begin{array}{l}\text { Conditions } \\
\text { of culture }\end{array}$} & \multicolumn{9}{|c|}{ Time of culture } \\
\hline & \multicolumn{5}{|c|}{$48 \mathrm{~h}$} & \multicolumn{4}{|c|}{2 weeks } \\
\hline & & $\mathrm{E}$ & $\mathrm{C}$ & $\mathrm{t}$ & p-value & $\mathrm{E}$ & $\mathrm{C}$ & $\mathrm{t}$ & p-value \\
\hline IFBS & $\begin{array}{r}5 \% \\
10 \%\end{array}$ & $\begin{array}{l}18.6 \pm 1.19 \\
20.3 \pm 0.35\end{array}$ & $\begin{array}{r}16.5 \pm 0.35 \\
15 \pm 0.32\end{array}$ & $\begin{array}{r}1.689 \\
11.051\end{array}$ & $\begin{array}{l}0.1421 \\
0.0000^{b}\end{array}$ & $\begin{array}{r}5 \pm 0.11 \\
4.2 \pm 0.11\end{array}$ & $\begin{array}{r}3.5 \pm 0.32 \\
2 \pm 0.23\end{array}$ & $\begin{array}{l}4.456 \\
8.521\end{array}$ & $\begin{array}{l}0.0043^{b} \\
0.0001^{b}\end{array}$ \\
\hline $\mathrm{pH}$ & $\begin{array}{l}7.4 \\
7.7 \\
8 \\
8.5\end{array}$ & $\begin{array}{c}11.3 \pm 0.33 \\
11.7 \pm 0.32 \\
11.4 \pm 0.28 \\
11 \pm 0.11\end{array}$ & $\begin{array}{l}13.6 \pm 0.25 \\
12.7 \pm 0.11 \\
13.5 \pm 0.29 \\
13.5 \pm 0.64\end{array}$ & $\begin{array}{l}-5.524 \\
-2.928 \\
-5.25 \\
-3.873\end{array}$ & $\begin{array}{l}0.0015^{b} \\
0.0264^{b} \\
0.0019^{b} \\
0.0082^{b}\end{array}$ & $\begin{array}{l}4.2 \pm 0.12 \\
5.3 \pm 0.12 \\
7.5 \pm 0.20 \\
7.3 \pm 0.14\end{array}$ & $\begin{array}{l}2.6 \pm 0.21 \\
2.5 \pm 0.18 \\
2.3 \pm 0.13 \\
2.5 \pm 0.24\end{array}$ & $\begin{array}{l}\quad 6.625 \\
12.962 \\
21.53 \\
17.527\end{array}$ & $\begin{array}{l}0.0006^{b} \\
0.0000^{b} \\
0.0000^{b} \\
0.0000^{b}\end{array}$ \\
\hline $\mathrm{CaCl}_{2} 1 \mathrm{mM}$ & & $23.2 \pm 0.45$ & $15.5 \pm 0.23$ & 15.199 & $0.0000^{b}$ & $18 \pm 0.15$ & $4.3 \pm 0.25$ & 47.458 & $0.0000^{b}$ \\
\hline $\begin{array}{l}\mathrm{MgCl}_{2} 1 \mathrm{mM} \\
\text { nutrient- } \\
\text { depleted culture }\end{array}$ & & $\begin{array}{l}30 \pm 0.36 \\
65 \pm 1.68\end{array}$ & $\begin{array}{l}15.3 \pm 0.47 \\
15.2 \pm 0.41\end{array}$ & $\begin{array}{l}24.789 \\
28.752\end{array}$ & $\begin{array}{l}0.0000^{b} \\
0.0000^{b}\end{array}$ & $\begin{array}{r}24.5 \pm 0.37 \\
6.2 \pm 0.15\end{array}$ & $\begin{array}{l}4.3 \pm 0.21 \\
1.9 \pm 0.23\end{array}$ & $\begin{array}{l}47.612 \\
15.879\end{array}$ & $\begin{array}{l}0.0000^{b} \\
0.0000^{b}\end{array}$ \\
\hline
\end{tabular}

$a$ : results as the mean values of four experiments \pm SEM; $t$ : observed $t$ statistic in a Independent-Sample T-Test, between experiments and controls results; $b$ : the difference between the experimental and that of the corresponding control was statistically significant, $\mathrm{p} \leq 0.05$; E: experiment; C: control.

Higher percentages were also observed in cultures where a greater quantity of IFBS was added (10\%), giving values of 20.3 vs $15 \%$ in control, which contained $2.5 \%$ IFBS. Supplementation of coccidial cultures with IFBS or other serum proteins affected coccidian motility and development (Upton et al. 1994).

Afterwards, the appropriate factors were selected and C. parvum was cultured in vivo by the following technique: the HCT-8 cells for which the culture medium had not been renewed for seven days were infected with C. parvum sporozoites in RPMI-1640 medium with $10 \%$ IFBS, pH 7.2 with $\mathrm{CaCl}_{2}$ and $\mathrm{MgCl}_{2} 1 \mathrm{mM}$, and were maintained with this medium for two weeks. The results (Table II) showed very high parasitism percentages $(71 \%$ in the experimental culture vs $14.5 \%$ in the control) which remained high after two weeks (47 vs 1.9\%). All the like cycle stages identified by Rosales et al. (2005) developed, both intracellular as well as extracellular, and the percentages of all these are reflected in Table II. Extracellular stages appeared after $72 \mathrm{~h}$ of in vitro culture, as indicated in other studies (Rosales et al. 1993, 2005, Hijjawi et al. 2002) in a low number in the controls $(2.1 \%$ at $72 \mathrm{~h}$ ) but the percentage of extracellular stages increased in the experimental cultures, peaking at $96 \mathrm{~h}(25.3 \%)$.

The origin of extracellular stages of $C$. parvum is not known, but it is possible that when we infected the culture with sporozoites, some penetrated the host cell and developed into circular trophozoites while other sporozoites developed extracellular motile trophozoite stages. Trophozoites of complementary types associated with each other to form syzygy and after this, formation of zygotes and sporogenesis occurred.

This in vitro culture technique for $C$. parvum in HCT 8 permits greater parasite development, raising the percentages of parasitized cells and of all the developmental phases of the parasite. This will enable better study of the extracellular stages or allow the use of these culture for therapeutic cultures.

\section{REFERENCES}

Becher KA, Robertson ID, Fraser DM, Palmer DG, Thompson RCA 2004. Molecular epidemiology of Giardia and Cryptosporidium infections in dairy calves originating from three sources in Western Australia. Vet Parasitol 123: 1-9.

Carreno RA, Schinitzler BE, Jeffries AC, Tenter AM, Johnson AM, Barta JR 1999. Cryptosporidium is more closely related to gregarines than to coccidian as shown by phylogenetic analysis of apicomplexan parasites inferred using small-subunit ribosomal RNA gene sequences. Parasitol Res 85: 899-904.

Entrala E, Molina JM, Rosales MJ, Sánchez M, Mascaró C 2000. Cryptosporidium parvum: oocysts purification using potassium bromide discontinuous gradient. Vet Parasitol 92: 223-226.

Fayer R, Morgan U, Upton SJ 2000. Epidemiology of Cryptosporidium: transmission, detection and identification. Inter J Parasitol 30: 1305-1322.

Griffin K, Matthai E, Hommel M, Weitz JC, Baxby D, Hart CA 1992. Antigenic diversity among oocysts of clinical isolates of Cryptosporidium parvum. J Protozool Res 2: 97-101.

Hamer DH, Ward H, Tzipori S, Pereira MEA, Alroy JP, Keusch GT 1994. Attachment of Cryptosporidium parvum sporozoites to MDCK cells in vitro. Infect Immun 62: 2208-2210.

Hijjawi NS 2003. In vitro cultivation and development of Cryptosporidium in cell culture. In RCA Thompson, A Armson, UM Morgan-Ryan, Cryptosporidium from Molecules to Disease, Elsevier, Amsterdam, p. 233-253.

Hijjawi NS, Meloni BP, Morgan UM, Thompson RCA 2001. Complete development and long-term maintenance of Cryptosporidium parvum human and cattle genotypes in cell cultures. Inter J Parasitol 31: 1048-1055.

Hijjawi NS, Meloni BP, Ng'anzo M, Ryan UM, Olson ME, Cox PT, Monis PT, Thompson RCA 2004. Complete development of Cryptosporidium parvum in host cell-free culture. Inter J Parasitol 34: 769-777.

Hijjawi NS, Meloni BP, Ryan UM, Olson ME, Thompson RCA 


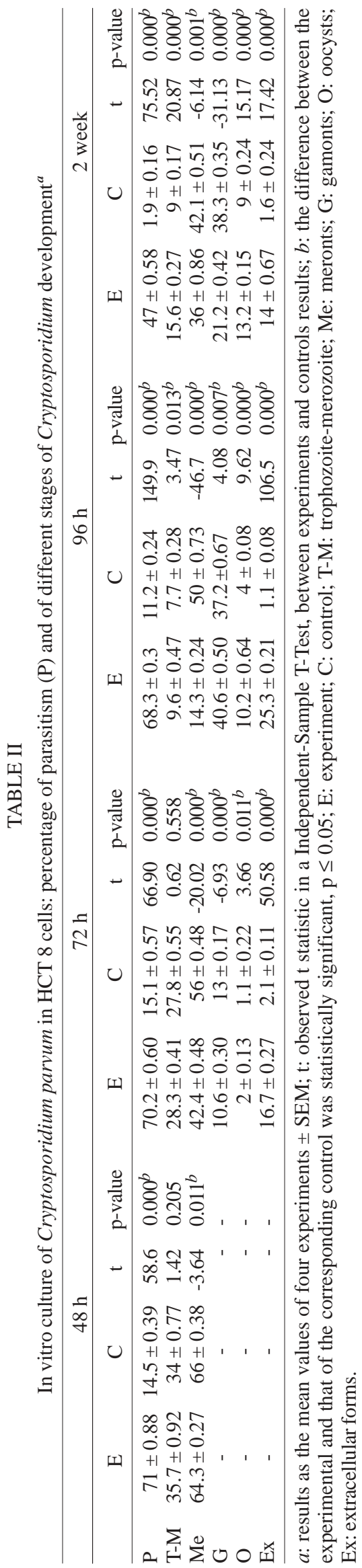

2002. Successful in vitro cultivation of Cryptosporidium andersoni: evidence for the existence of novel extracellular stages in the life cycle and implications for the classification of Cryptosporidium. Intern J Parasitol 32: 1719-1726.

Lacharme L, Villar V, Rojo-Vazquez FA, Suárez S 2004. Complete development of Cryptosporidium parvum in rabbit chondrocytes (VELI cells). Microbes Infection 6: 566-571.

Leander BS, Clopton RE, Keeling PJ 2003. Phylogeny of gregarines (Apicomplexa) as inferred from small-subunitrDNA and beta-tubulin. Int J Syst Evol Microbiol 53: 345-354.

Maillot C, Favennec L, Francois A, Ducrotte P, Brasseur PJ 1997. Sexual and asexual development of Cryptosporidium parvum in five oocysts or sporozoite infected human enterocytic cell lines. Eukaryot Microbiol 44: 582-585.

McDonald V, Deer RMA, Nina JMS, Wright S, Chiodini PL, McAdam KPWJ 1991. Characterization and specificity of hybridoma antibodies against oocyst antigens of Cryptosporidium parvum from man. Parasite Immunol 13: 251-259

Monis PT, Thompson RCA 2003. Cryptosporidium and Giardia-zoonoses: fact or fiction? Infect Gen Evol 3: 233-244.

Pérez-Cordón G, Rosales MJ, Sánchez-Moreno M 2005. Processing of fecal simples for study of Cryptosporidium sp. by PCR. Rev Peru Biol 12: 158-160.

Ramirez E, Ward LA, Sreevatsan S 2004. A review of the biology and epidemiology of cryptosporidiosis in humans and animals. Microbes Infection 6: 773-785.

Robertson LJ, Campbell AT, Smith HV 1993. In vitro excystation of Cryptosporidium parvum. Parasitology 106: 13-19.

Rosales MJ, Cifuentes J, Mascaró C 1993. Cryptosporidium parvum: culture in MDCK cells. Exper Parasitol 76: 209-212.

Rosales MJ, Mascaró C, Arnedo T, Castilla JJ 1994. Isolation and identification of Cryptosporidium parvum oocysts with continuous percoll gradients and combined alcian blue-giemsa staining. Acta Trop 56: 371-373.

Rosales MJ, Peréz-Cordón G, Sánchez Moreno M, Marín Sánchez C 2005. Extracellular like-gregarine stages of Cryptosporidium parvum. Acta Trop 95: 74-78.

Slapeta J 2006. Cryptosporidium species found in cattle: a proposal for a new species. Trends Parasitol 22: 469-474.

Upton SJ, Tilley M, Brillhart DB 1994. Comparative development of Cryptosporidium parvum (Apicomplexa) in 11 continous host cell lines. FEMS Microbiol Lett 118: 233-236.

Xiao L, Escalante L, Yang C, Sulaiman I, Escalante A, Montali R, Fayer R, Altaf A 1999a. Phylogenetic analysis of Cryptosporidium parasites based on the small subunit rRNA gene locus. Appl Environ Microbiol 65: 1578-1583.

Xiao L, Morgan U, Limor J, Escalante A, Arrowood M, Shulaw W, Thompson R, Fayer R Altaf A 1999b. Genetic diversity within Cryptosporidium parvum and related Cryptosporidium species. Appl Environ Microbiol 65: 3386-3391.

Ward LA, Wang Y 2001. Rapad methods to isolate Cryptosporidium from frozen feces for PCR. Diag Microbiol Infec Dis 41: 37-42.

Yang S, Healey MC, Chunwei Zhang J 1996. Complete development of Cryptosporidium parvum in bovine fallopian tube epithelial cells. Infect Immun 64: 349-354. 
